\title{
Lactic Acidosis in Diabetic Population: Is Metformin Implicated? Results of a Matched Case-Control Study Performed on the Type 2 Diabetes Population of Grenoble Hospital University
}

\author{
Marion Lepelley, ${ }^{1}$ Joris Giai, ${ }^{2,3}$ Nassima Yahiaoui, ${ }^{1}$ \\ Sébastien Chanoine, ${ }^{4,5}$ and Céline Villier ${ }^{1}$ \\ ${ }^{1}$ Centre Régional de Pharmacovigilance, Pôle Santé Publique, Centre Hospitalier Universitaire de Grenoble, 38000 Grenoble, France \\ ${ }^{2}$ Service de Biostatistique des Hospices Civils de Lyon, 69002 Lyon, France \\ ${ }^{3}$ Laboratoire Biostatistique Santé, UCBL, Equipe de l'UMR CNRS 5558, 69495 Pierre-Bénite Cedex, France \\ ${ }^{4}$ Pôle Pharmacie, Centre Hospitalier Universitaire de Grenoble, 38000 Grenoble, France \\ ${ }^{5}$ Université Grenoble Alpes, 38000 Grenoble, France
}

Correspondence should be addressed to Marion Lepelley; mlepelley@chu-grenoble.fr

Received 10 December 2015; Revised 15 January 2016; Accepted 8 February 2016

Academic Editor: Mitsuhiko Noda

Copyright (C) 2016 Marion Lepelley et al. This is an open access article distributed under the Creative Commons Attribution License, which permits unrestricted use, distribution, and reproduction in any medium, provided the original work is properly cited.

\begin{abstract}
Introduction. To evaluate the strength of association between lactic acidosis (LA) and well-recognized risk factors for LA, particularly the weight of metformin. Methods. This study is a matched case-control analysis concerning the type 2 diabetes population from Grenoble Hospital University. Cases of LA were defined biologically with $\mathrm{pH}<7.35$ and lactates $>5 \mathrm{mmol} / \mathrm{L}$. They were matched to 2 controls defined as type 2 diabetic inpatients who did not present a LA during the study period. We performed a conditional logistic regression. Results. We included 302 cases and 604 controls; mean age was 69.5 years (SD 11.93). Intercurrent diseases were significantly associated with LA. Chronic medical conditions had a minor impact on LA incidence, except hepatocellular dysfunction. Metformin was significantly associated with a higher LA probability in case of acute kidney injury $(\mathrm{AKI})(\mathrm{OR}=1.79$; $p$ value $=0.020)$ but not in patients without AKI. Discussion and Conclusions. According to this study, metformin, compared to acute medical conditions, seemed not to be associated with LA in patients with type 2 diabetes; however in case of AKI, metformin may be associated with LA.
\end{abstract}

\section{Introduction}

According to the World Health Organization, diabetic population should reach almost 600 million worldwide, in 2035 [1]. When medication is required, metformin is the first-line treatment in type 2 diabetes due to its superiority in reducing cardiovascular events and morbidity-mortality, compared to insulin and sulfonylureas [2-4].

The safety profile of metformin is well established: gastrointestinal disorders are the most frequent adverse effect. Lactic acidosis (LA) is a very rare but potentially severe adverse effect resulting from hepatic gluconeogenesis and mitochondrial respiration inhibition.

Causality between metformin and the occurrence of LA is a matter for debate and remains controversial. According to several studies, the risk of developing a LA due to metformin exposure is low, ranging from 2 to 9 cases per 100000 patientyears [5-8]. A review of prospective trials and observational cohort studies did not find an increased risk of LA with metformin compared to other antihyperglycemic treatments [7]. A nested case-control analysis did not find any difference concerning occurrence of LA between metformin and sulfonylureas groups but identified risk factors for LA in all case subjects [8]. Furthermore, many cases of LA are reported in patients treated with metformin when presenting acute kidney failure due to dehydration or exposure to nephrotoxic drugs such as nonsteroidal anti-inflammatory drugs (NSAID), diuretics, and angiotensin converting enzyme (ACE) inhibitor. The spontaneous reporting of such cases is currently growing. 
The mortality associated with this rare adverse drug effect is $50 \%$ with all biguanides [9]. Recently, it was estimated to be around 26 to $30 \%$ with metformin $[5,10]$. When measured, plasmatic level of metformin is difficult to interpret; its correlation with lactate levels has not been demonstrated [7]. In case of LA, metformin level is significantly higher in survivors than in nonsurvivors [11].

This apparent discrepancy between clinical trials and real life probably results from the presence of uncontrolled intercurrent diseases.

Most contraindications, special warnings, and precautions for use labelled in the metformin summary product characteristics are aimed at minimizing this risk. Recently, contraindications have been judged unjustified and too restrictive [12]. For example, metformin is more and more prescribed on the basis of studies in moderate chronic renal failure or in case of cardiac failure [13-16]. It has been estimated that around half the patients would be deprived of this useful drug by a strict application of recommendations $[13,17,18]$. The potential consequence of such a change in prescription pattern is that intercurrent diseases could lead to more severe LA.

In this context, we conducted a study to evaluate the strength of association between the occurrence of LA in patients suffering type 2 diabetes and the well-recognized risk factors for LA and particularly to determine the impact of a metformin treatment among all these factors. These risk factors for LA were related to an underlying chronic condition or to an intercurrent acute disease.

\section{Methods}

We performed a matched case-control study on the type 2 diabetic population from Grenoble University Hospital, between 1 January 2008 and 31 December 2011.

2.1. Population, Cases, and Controls. The source population included every inpatient with type 2 diabetes admitted at Grenoble University Hospital between 2008 and 2011, according to the 10th Edition of the International Classification of Diseases (ICD). Considering potential coding approximations, we selected 3 ICD codes: non-insulin-dependent diabetes mellitus (E11), other specified diabetes mellitus (E13), and unspecified diabetes mellitus (E14). To exclude potential type 1 diabetic patients, we rejected patients under 30 years of age.

Every type 2 diabetic inpatient who presented a LA between 2008 and 2011 was considered as a potential case. According to Cohen and Wood's definition, LA was defined biologically as a metabolic acidosis characterized by an arterial $\mathrm{pH}<7.35$ and a lactate level $>5 \mathrm{mmol} / \mathrm{L}$ [19]. Group control included type 2 diabetic inpatients who did not present a LA during the study period. We decided to match one case to two controls, considering the rareness of controls with extreme ages. Our choice was to keep all cases included in the study.

Two controls were matched to every case based on gender, year of birth, and date of hospitalization. When more than two controls were available for matching, the choice was made at random. The case and its matched controls were excluded in case of missing data or ICD coding errors.

2.2. Potential Risk Factors and Exposure to Metformin. The list of LA risk factors was established based on the CohenWoods classification [19], on the analysis of case-reports in the literature and spontaneous reports of LA in the French national pharmacovigilance database. We distinguished chronic medical conditions, concomitant therapies, and intercurrent diseases.

Exposure to metformin and potential risk factors for LA were sought in every computerized medical record shortly before the date of diagnosis of LA. When necessary, we checked results of laboratory exams to be quite sure intercurrent diseases were present before $\mathrm{pH}$ and lactates became pathologic. Fatal outcome was also investigated for every patient.

Chronic medical conditions investigated were chronic kidney disease (CKD), hepatocellular dysfunction, chronic respiratory failure, chronic heart failure, neoplasia, mitochondrial dysfunction, and pyridoxine deficit.

Concomitant therapies investigated were ACE inhibitors, angiotensin II receptor antagonists (ARA), diuretics, antiretroviral (ARV) drugs, NSAID, metformin, insulin, and iodinated contrast media (CM).

Intercurrent diseases investigated were shock (cardiac, hypovolemic, and septic), severe anemia, dehydration (diarrhea, vomiting), acute kidney injury (AKI), acute hepatic failure, acute respiratory failure, myocardial infarction, acute decompensated heart failure, sepsis, convulsions, intense muscular effort, and acute artery occlusion.

2.3. Statistical Analysis. Statistical analyses were performed using STATA software (version 11.0, STATA Corp, College Station, Texas). Quantitative data were displayed as means and standard deviations (SD). Qualitative data were expressed with number of subjects and percentages. In case of weak effective, variables were excluded or grouped. The alpha risk was set at $5 \%$.

2.3.1. Univariate Analysis. We performed McNemar chisquared tests and Student's tests for matched data. Covariates with low effectives $(<5 \%)$ were excluded. Covariates were included for further analysis if $p$ value $<0.25$. Multicollinearity analysis of all covariates was performed.

2.3.2. Multivariate Analysis. We used a model backward elimination process for the conditional logistic regression. After each step, a likelihood-ratio test was performed to compare models. Metformin was forced in the model despite its nonsignificance.

Interactions were searched in the model of conditional logistic regression. When an interaction was found, we achieved logistic regression with stratification.

A Hosmer-Lemeshow test was achieved for the final multivariate model. A model is considered suitable if the $p$ value of this test exceeds $5 \%$. 


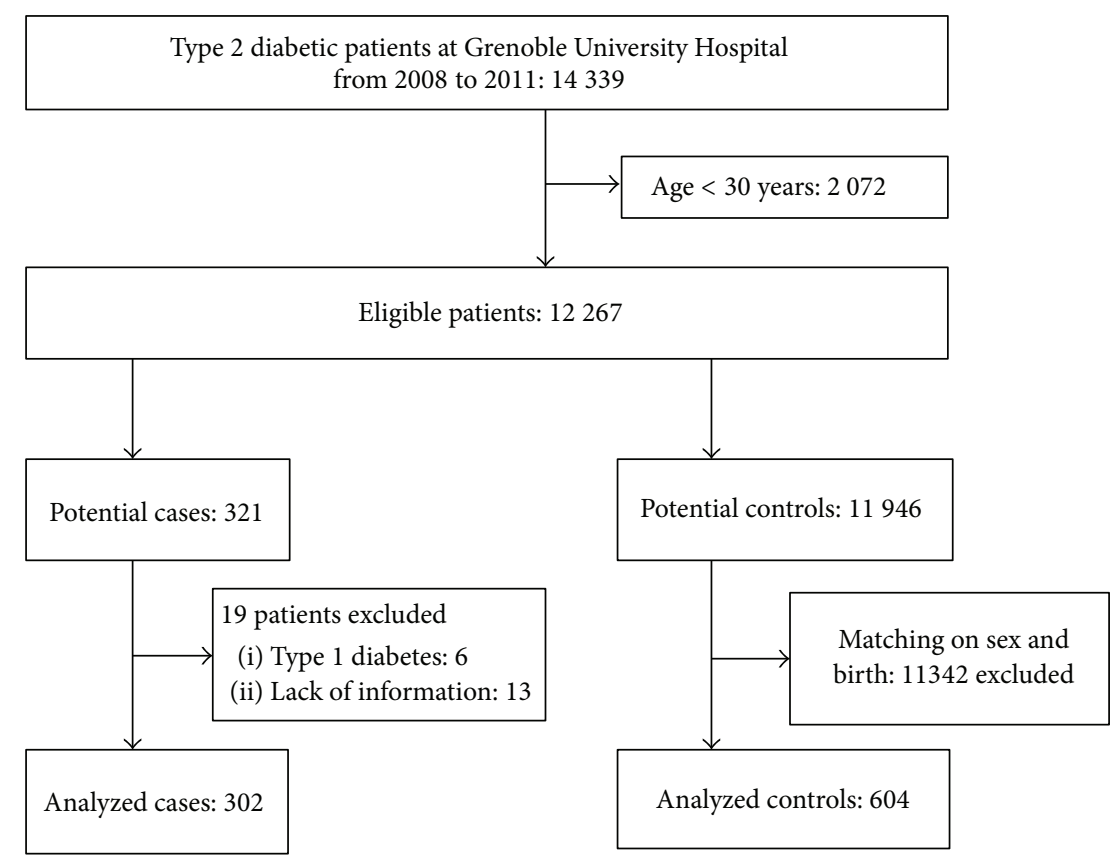

FIGURE 1: Study flow chart.

In order to assess the goodness of fit of the final model, we estimated its discriminatory power by using Receiver Operator Characteristic (ROC) curve and by calculating its sensitivity and specificity. Goodness of fit is considered to be acceptable if the area under the ROC curve is comprised between 0.7 and 0.8 , good between 0.8 and 0.9 , excellent above 0.9 .

\section{Results}

3.1. Population Study. Initial list of type 2 diabetic patients contained a large number of patients, more than 14000 . After application of exclusion criteria, our source population included 12267 eligible subjects. The flow chart of our study is presented in Figure 1.

3.2. Cases and Controls. The number of cases, that is, type 2 diabetic patients who presented a LA during 2008 and 2011, was 321. Nineteen cases were excluded because of coding errors (6 patients suffering type 1 diabetes) or lack of information (13 patients). Thus, a total of 302 cases were analyzed. They were matched to 604 controls. The final population study included 906 patients (Table 1).

There was no difference between cases and controls regarding age and sex, according to the study design; most chronic medical conditions: existence of CKD, mitochondrial dysfunction, neoplasia, or pyridoxine deficit; and most concomitant therapies: ACE inhibitors, ARA, diuretics, ARV drugs, and insulin.

Cases were significantly more affected by hepatic, cardiac, respiratory chronic diseases or advanced renal failure; more exposed to NSAID and iodinated CM; and more affected by any intercurrent disease. Mortality was logically higher among cases.
Controls were significantly more treated by metformin than cases.

\subsection{Strength of Association between Covariates and Occurrence of $L A$}

3.3.1. Univariate Analysis. Due to weak effective ( $<5 \%)$, for further analyses we included the following covariates among chronic conditions: CKD, hepatocellular dysfunction, chronic respiratory failure, heart failure, and neoplasia; among concomitant therapies: ACE inhibitors, ARA, diuretics, metformin, insulin, and iodinated CM; and among intercurrent diseases: AKI, acute respiratory failure, acute heart failure, and sepsis. We defined 2 groups of CKD (instead of 4), namely, mild-moderate and severe-end stage.

According to univariate analysis, among chronic medical conditions and concomitant therapies, only hepatocellular dysfunction, chronic respiratory failure, heart failure, and NSAID were associated with a higher rate of LA. All intercurrent diseases were significantly associated with LA. Metformin treatment was the only factor which seems to be protective (odds ratio $(\mathrm{OR})=0.68$; CI 95\%: [0.53-0.86], result not shown).

Neoplasia and insulin were excluded due to $p$ value $\geq$ 0.25 . Diuretics were kept in the analysis despite a $p$ value $=$ 0.264 . Metformin was kept too, despite its apparent protective effect.

3.3.2. Multivariate Analysis. According to our final model (Table 2), among chronic medical conditions, only hepatocellular dysfunction was associated with LA. Surprisingly, early CKD, mild and moderate stage, seemed to have a protective effect. No concomitant therapy was associated with 
TABLE 1: Demographic characteristics and comparative analysis of covariates $(N=906)$.

\begin{tabular}{|c|c|c|c|c|c|}
\hline & \multicolumn{2}{|c|}{ Cases $(n=302)$} & \multicolumn{2}{|c|}{ Controls $(n=604)$} & \multirow{2}{*}{$p$ value } \\
\hline & $n$ & $(\%)$ & $n$ & $(\%)$ & \\
\hline \multicolumn{6}{|l|}{ Demographic characteristics } \\
\hline Age, mean (SD) & $69.4(11.90)$ & - & $69.5(11.90)$ & - & 0.714 \\
\hline Male & 177 & $(58.6)$ & 354 & $(58.6)$ & 1 \\
\hline \multicolumn{6}{|l|}{ Chronic medical conditions } \\
\hline CKD & 95 & $(31.5)$ & 224 & $(37.1)$ & 0.094 \\
\hline Mild-moderate & 66 & $(21.9)$ & 184 & $(30.5)$ & \multirow{2}{*}{0.013} \\
\hline Severe-end stage & 29 & $(9.6)$ & 40 & (6.3) & \\
\hline Hepatocellular dysfunction & 47 & $(15.6)$ & 39 & $(6.5)$ & $<0.001$ \\
\hline Chronic respiratory failure & 84 & $(27.8)$ & 127 & $(21.0)$ & 0.023 \\
\hline Heart failure & 82 & $(27.2)$ & 94 & $(15.6)$ & $<0.001$ \\
\hline Mitochondrial dysfunction & 2 & $(0.7)$ & 0 & $(0)$ & 0.111 \\
\hline Neoplasia & 38 & $(12.6)$ & 81 & $(13.4)$ & 0.728 \\
\hline Pyridoxine deficit & 3 & $(1.0)$ & 13 & $(2.2)$ & 0.288 \\
\hline \multicolumn{6}{|l|}{ Concomitant therapies } \\
\hline ACE inhibitors & 81 & $(26.8)$ & 186 & $(30.8)$ & 0.216 \\
\hline ARA & 65 & $(21.5)$ & 155 & $(25.7)$ & 0.171 \\
\hline Diuretics & 150 & $(49.7)$ & 321 & $(53.2)$ & 0.323 \\
\hline ARV & 1 & $(0.3)$ & 5 & $(0.8)$ & 0.670 \\
\hline NSAID & 14 & $(4.6)$ & 12 & $(2.0)$ & 0.024 \\
\hline Metformin & 111 & $(36.8)$ & 276 & $(45.7)$ & 0.010 \\
\hline Insulin & 129 & $(42.7)$ & 268 & $(44.4)$ & 0.636 \\
\hline Iodinated CM & 37 & $(12.3)$ & 17 & (2.8) & $<0.001$ \\
\hline \multicolumn{6}{|l|}{ Intercurrent diseases } \\
\hline Shock & 149 & $(49.3)$ & 8 & (1.3) & $<0.001$ \\
\hline Severe anemia & 57 & $(18.9)$ & 19 & $(3.2)$ & $<0.001$ \\
\hline Dehydration & 35 & $(11.6)$ & 17 & (2.8) & $<0.001$ \\
\hline AKI & 184 & $(60.9)$ & 80 & $(13.3)$ & $<0.001$ \\
\hline Acute hepatic failure & 64 & $(21.2)$ & 12 & $(2.0)$ & $<0.001$ \\
\hline Acute respiratory failure & 156 & $(51.7)$ & 41 & $(6.8)$ & $<0.001$ \\
\hline Myocardial infarction & 32 & $(10.6)$ & 16 & $(2.7)$ & $<0.001$ \\
\hline Acute decompensated heart failure & 130 & $(43.1)$ & 45 & $(7.5)$ & $<0.001$ \\
\hline Sepsis & 134 & $(44.4)$ & 49 & $(8.1)$ & $<0.001$ \\
\hline Convulsions & 21 & $(7.0)$ & 8 & $(1.3)$ & $<0.001$ \\
\hline Intense muscle effort & 6 & $(2.0)$ & 0 & - & 0.001 \\
\hline Acute artery occlusion & 30 & $(9.9)$ & 28 & $(4.6)$ & 0.002 \\
\hline Death & 146 & $(48.3)$ & 26 & $(4.3)$ & $<0.001$ \\
\hline
\end{tabular}

LA occurrence. After adjustment on other risk factors, metformin could not be considered anymore as a protective factor. All the intercurrent diseases that could have been included in the analysis were significantly associated with LA.

Interaction analysis showed that AKI interacted with metformin. We stratified our population on the basis of occurrence of AKI.

Overall, 264 patients presented an AKI, mostly cases (Table 3 ). There were no differences concerning sex ratio $(p=0.993)$. Proportion of deaths was higher among cases and they were more exposed to metformin than controls but metformin was not significant according to univariate analysis $(\mathrm{OR}=1.51$; CI 95\%: [0.84-2.77]). In multivariate analysis (Table 4), variables significantly associated with LA in patients who presented an AKI were shock, acute respiratory failure, injection of iodinated CM, severe anemia, hepatocellular dysfunction, acute decompensated heart failure, sepsis, and metformin. Early stage CKD (mild and moderate) was considered as a protective factor. According to Hosmer and Lemeshow test, this model fitted well the data $(p=0.482)$. Metformin was a significant risk factor for LA in presence of AKI.

On the other side, 642 patients did not present an AKI. There were more controls than cases (Table 3 ). There was no significant difference of age $(p=0.573)$ and sex ratio. We observed more deaths in the case group. Controls were treated more often with metformin than cases. In univariate analysis, metformin was a protective factor $(\mathrm{OR}=0.48$; CI 
TABLE 2: Conditional logistic regression of covariates $(N=906)$.

\begin{tabular}{|c|c|c|c|}
\hline Variables & Matched OR & CI 95\% & $p$ value \\
\hline \multicolumn{4}{|l|}{ Chronical medical condition } \\
\hline No CKD & 1 & - & - \\
\hline CKD mild-moderate & 0.36 & {$[0.19-0.69]$} & 0.002 \\
\hline CKD severe-end stage & 1.62 & {$[0.62-4.25]$} & 0.325 \\
\hline Hepatocellular dysfunction & 6.51 & {$[2.78-15.25]$} & $<0.001$ \\
\hline \multicolumn{4}{|l|}{ Concomitant therapies } \\
\hline Metformin & 1.27 & {$[0.73-2.22]$} & 0.390 \\
\hline \multicolumn{4}{|l|}{ Intercurrent diseases } \\
\hline AKI & 9.58 & {$[5.24-17.47]$} & $<0.001$ \\
\hline Acute respiratory failure & 9.34 & {$[4.76-18.32]$} & $<0.001$ \\
\hline Acute decompensated heart failure & 3.55 & {$[1.84-6.84]$} & $<0.001$ \\
\hline Sepsis & 8.28 & [4.28-15.99] & $<0.001$ \\
\hline
\end{tabular}

95\% [0.30-0.74]). In multivariate analysis (Table 4), acute respiratory failure, sepsis, acute decompensated heart failure, and hepatocellular dysfunction were significantly associated with occurrence of LA in patients without AKI. Early CKD stage was a protective factor for LA $(\mathrm{OR}=0.33 ; p=0.003)$. Hosmer and Lemeshow test indicated a good fit $(p=0.416)$. Metformin was no longer associated with LA in type 2 diabetic patients without acute renal dysfunction $(\mathrm{OR}=0.86$; $p=0.628)$.

3.3.3. Fit of the Final Model. The maximum likelihood $R^{2}$ of our model was 0.878 . The predictive accuracy of our model was calculated: sensitivity was $82.78 \%$ and specificity was $94.20 \%$. Area under the curve (AUC) is estimated at 0.83 .

\section{Discussion}

Several studies on risk factors significantly associated with LA are published but none evaluated the relative importance of these risk factors. This work showed a significant statistical association between a chronic hepatocellular dysfunction, a sepsis, an acute renal, respiratory, or cardiac disease, and occurrence of LA in type 2 diabetic patients.

We performed a backward elimination approach for the multivariable conditional logistic regression model. The method used to choose variables to be included in such model has always been of great concern in epidemiological studies. Backward elimination has the advantage of being objective and its major drawback is the likeliness of real risk factors exclusion. Thus, in addition to likelihood-ratio tests, we made sure at each selection step that the remaining OR did not vary very much. We also informally compared effect sizes between the full model and the final one.

Our results suggested that chronic medical conditions had a minor impact on LA incidence. Hepatocellular dysfunction is the only chronical medical condition significantly associated with LA. This model showed that end or severe stage of CKD (clearance $<30 \mathrm{~mL} / \mathrm{min}$ ) per se is not a risk factor for LA. Early and mild stages of CKD (clearance between 89 and $30 \mathrm{~mL} / \mathrm{min}$ ) even provide protection. This result is constantly found in all the multivariate interaction's analysis.
ACE, ARA, NSAID, diuretics, and iodinated CM have the potential to cause LA indirectly by acute renal failure; ARV drugs by direct action on mitochondrial activity. However they did not appear in the final model. One explanation could be that they have been stopped just before hospitalization.

Regarding metformin, controls were significantly more often treated with metformin $(45.70 \%)$ than cases $(36.75 \%)$ $(p$ value $=0.015)$. This can be explained by the fact that contraindications were relatively well respected. In our study, only 5 cases $(1.65 \%)$ and 1 control $(0.16 \%)$ with severe and end stage CKD were treated with metformin.

Numerous studies suggested that metformin was safe in patients with moderate renal impairment [20-22]. Since January 2013, metformin is allowed at a dosage of $1.5 \mathrm{~g}$ per day in France in case of glomerular filtration rate (GFR) comprised between 30 and $60 \mathrm{~mL} / \mathrm{min}$ [2]. Guidelines from the NICE published in 2008 suggested that metformin should be reviewed at GFR $=45 \mathrm{~mL} / \mathrm{min}$ and stopped at 30 [23].

In this study, intercurrent diseases were significantly associated with LA. A study of every LA cause in an English hospital found that most frequent precipitating factors for LA in type 2 diabetic patients were cardiac and respiratory decompensation, AKI, and sepsis [24]. Patients with type 2 diabetes have a 2.5-fold increased risk of AKI, compared to nondiabetic patients [25]. AKI is the most often encountered comorbidity in association with LA $[6,24,26]$.

We paid great attention not to include potential confounding factors such as secondary organ insufficiency in the model. When LA was diagnosed lately, we may have included potential outcomes of LA. It can be a potential bias to our study that we try to minimize by the careful study of all medical records.

We identified an interaction between metformin and AKI: metformin was significantly associated with occurrence of LA in case of AKI $(\mathrm{OR}=1.79 ; p$ value $=0.020)$ but not in patients without AKI. This later result is consistent with what is observed in daily practice or in spontaneous reporting to the pharmacovigilance system and is probably linked to an accumulation of metformin in organism. Injection of iodinated CM is also a potential risk factor in patients with AKI. Thereby, metformin discontinuation before injection of 
TABLE 3: Descriptive analysis of patients with $(n=264)$ and without $(n=642)$ AKI.

\begin{tabular}{|c|c|c|c|c|}
\hline \multirow{2}{*}{ Variables } & \multicolumn{2}{|c|}{$\begin{array}{c}\text { Patients with an AKI } \\
n=264\end{array}$} & \multicolumn{2}{|c|}{$\begin{array}{l}\text { Patients without an AKI } \\
\qquad n=642\end{array}$} \\
\hline & $\begin{array}{l}\text { Cases }(\%) \\
(n=184)\end{array}$ & $\begin{array}{l}\text { Controls }(\%) \\
\quad(n=80)\end{array}$ & $\begin{array}{l}\text { Cases }(\%) \\
(n=118)\end{array}$ & $\begin{array}{c}\text { Controls }(\%) \\
(n=524)\end{array}$ \\
\hline \multicolumn{5}{|l|}{ Demographic characteristics } \\
\hline Age, mean (SD) & $70.34(11.24)$ & $74.21(11.55)$ & $68.06(13.06)$ & $68.75(11.85)$ \\
\hline Male & $108(58.7)$ & $47(58.8)$ & 49 (41.5) & $217(41.4)$ \\
\hline \multicolumn{5}{|l|}{ Chronic medical condition } \\
\hline CKD & $66(35.9)$ & $52(65.0)$ & $29(24.6)$ & $172(32.8)$ \\
\hline Mild-moderate & $47(25.5)$ & $42(52.5)$ & $19(65.5)$ & $142(82.6)$ \\
\hline Severe-end stage & $19(10.3)$ & $10(12.5)$ & $10(34.5)$ & $30(17.4)$ \\
\hline Hepatocellular dysfunction & $32(17.4)$ & $7(8.8)$ & $15(12.7)$ & $32(6.1)$ \\
\hline Chronic respiratory failure & $45(24.5)$ & $22(27.5)$ & $39(33.1)$ & $105(20.0)$ \\
\hline Heart failure & $47(25.5)$ & $26(32.5)$ & $35(29.7)$ & $68(13.0)$ \\
\hline Neoplasia & 0 & $1(0.5)$ & $21(17.8)$ & $70(13.4)$ \\
\hline Mitochondrial dysfunction & $17(9.2)$ & $11(13.8)$ & $1(0.9)$ & 0 \\
\hline Pyridoxine deficit & $2(1.1)$ & $1(1.3)$ & $1(0.9)$ & $12(2.3)$ \\
\hline \multicolumn{5}{|l|}{ Concomitant therapies } \\
\hline ACE inhibitors & $50(27.2)$ & $34(42.5)$ & $31(26.3)$ & $152(29.0)$ \\
\hline ARA & $45(24.5)$ & $20(25.0)$ & $20(17.0)$ & $135(25.8)$ \\
\hline Diuretics & $95(51.6)$ & $49(61.3)$ & $55(46.6)$ & $272(51.9)$ \\
\hline ARV & $1(0.5)$ & $1(1.3)$ & 0 & $4(0.8)$ \\
\hline NSAID & $11(6.0)$ & $1(1.3)$ & $3(2.5)$ & $11(2.1)$ \\
\hline Metformin & $75(40.8)$ & $25(31.3)$ & $36(30.5)$ & $251(47.9)$ \\
\hline Insulin & $73(39.7)$ & $40(50.0)$ & $56(47.5)$ & $228(43.5)$ \\
\hline Iodinated CM & $20(10.9)$ & $5(6.3)$ & $17(14.4)$ & $12(2.3)$ \\
\hline \multicolumn{5}{|l|}{ Intercurrent diseases } \\
\hline Shock & $112(60.9)$ & $6(7.5)$ & $37(31.4)$ & $2(0.4)$ \\
\hline Severe anemia & $38(20.7)$ & $5(6.3)$ & $19(16.1)$ & $14(2.7)$ \\
\hline Dehydration & $30(16.3)$ & $10(12.5)$ & $5(4.3)$ & $7(1.3)$ \\
\hline Acute hepatic failure & $55(29.9)$ & $3(3.8)$ & $9(7.6)$ & $7(1.7)$ \\
\hline Acute respiratory failure & $94(51.1)$ & $12(15.0)$ & $62(52.2)$ & $29(5.5)$ \\
\hline Myocardial infarction & $21(11.4)$ & $5(6.3)$ & $11(9.3)$ & $11(2.1)$ \\
\hline Acute decompensated heart failure & $80(43.5)$ & $18(22.5)$ & $50(42.4)$ & $27(5.2)$ \\
\hline Sepsis & $93(50.5)$ & $13(16.3)$ & $41(34.8)$ & $36(6.9)$ \\
\hline Convulsions & $10(5.4)$ & $3(3.8)$ & $11(9.3)$ & $5(1.0)$ \\
\hline Intense muscle effort & $2(1.1)$ & 0 & $4(3.4)$ & 0 \\
\hline Acute artery occlusion & $23(12.5)$ & $4(5.0)$ & $7(5.9)$ & $24(4.6)$ \\
\hline Death & $94(51.1)$ & $10(12.5)$ & $52(44.1)$ & $16(3.1)$ \\
\hline
\end{tabular}

any iodinated CM and biological follow-up of renal function are valuable measures.

The width of confidence intervals for hepatocellular dysfunction, AKI, acute respiratory failure, and sepsis highlighted weak effectives. Indeed, 302 cases could be considered as insufficient to precisely weight risk factors for a rare event. But we were not able to get the data before 2008 because they were not computerized. If such a study should be repeated, it would be in a bigger hospital and within a longer time frame. We chose to match one case to two controls. Matching to more than two controls would have increased power of our study. However we could not match to more than two controls due to low number of patients at extreme ages. A solution could be to realize this study in a larger sample.

A probably useful parameter to consider would have been the advanced stage of diabetes. Micro- and macrovascular complications can lead to more hypoxemia and chronic hyperglycemic and/or hypoinsulinic state to a more important glucose load. Unfortunately information of the disease was often missing in the electronic medical records, since 
TABLE 4: Multivariate analysis with stratification variable: AKI.

\begin{tabular}{|c|c|c|c|c|c|c|}
\hline \multirow[t]{2}{*}{ Variables } & \multicolumn{3}{|c|}{$\begin{array}{c}\text { Patients with AKI } \\
n=264\end{array}$} & \multicolumn{3}{|c|}{$\begin{array}{l}\text { Patients without AKI } \\
\qquad n=642\end{array}$} \\
\hline & OR & CI 95\% & $p$ value & $\mathrm{OR}$ & CI 95\% & $p$ value \\
\hline \multicolumn{7}{|l|}{ Chronic medical condition } \\
\hline CKD mild-moderate & 0.42 & {$[0.25-0.73]$} & 0.002 & 0.33 & {$[0.16-0.68]$} & 0.003 \\
\hline CKD severe-end stage & 1.82 & {$[0.80-4.13]$} & 0.149 & 1.29 & {$[0.49-3.43]$} & 0.607 \\
\hline Hepatocellular dysfunction & 5.29 & {$[2.56-10.95]$} & $<0.001$ & 5.17 & {$[2.29-11.67]$} & $<0.001$ \\
\hline \multicolumn{7}{|l|}{ Concomitant therapies } \\
\hline Metformin & 1.79 & [1.09-2.93] & 0.020 & 0.86 & {$[0.48-1.55]$} & 0.628 \\
\hline Iodinated CM & 8.58 & [3.77-19.52] & $<0.001$ & - & - & - \\
\hline \multicolumn{7}{|l|}{ Intercurrent diseases } \\
\hline Shock & 42.06 & [17.91-98.78] & $<0.001$ & - & - & - \\
\hline Severe anemia & 5.83 & {$[2.58-3.17]$} & $<0.001$ & - & - & - \\
\hline Acute respiratory failure & 11.86 & {$[6.79-20.74]$} & $<0.001$ & 12.38 & {$[6.52-23.50]$} & $<0.001$ \\
\hline Acute decompensated heart failure & 5.19 & {$[2.88-9.36]$} & $<0.001$ & 6.29 & {$[3.12-12.66]$} & $<0.001$ \\
\hline Sepsis & 4.34 & {$[2.45-7.70]$} & $<0.001$ & 6.87 & [3.61-13.07] & $<0.001$ \\
\hline
\end{tabular}

some patients arrived at Grenoble University Hospital for the first time through emergency or intensive care units. Similarly, we could not differentiate the severity of chronic hepatocellular dysfunction based on value of prothrombin ratio.

The mean age of diabetic population in France is 65 [27]. The mean age of our sample is around 69.5. This could be explained by the hospital recruitment: diabetic patients followed up in hospital or requiring a hospitalization may have a more severe condition than those followed up by private practitioners or who do not need hospitalization. Age may influence comorbidities, clinical condition, and the need for more complex care.

Case and control groups were predominantly men; this fact is comparable to the masculine sex ratio described in data from the French National Institute for Public Health Surveillance [27]. There was no sex difference concerning occurrence of LA.

Mortality was much higher among cases $(48.3 \%)$ than controls (4.3\%). Our mortality rate of cases is consistent with previous studies in literature: mortality rate with all biguanides was estimated around 50\% [9], even though it was reduced to $26-30 \%$ with metformin only $[5,10]$.

Considering treatment with ACE, ARA, thiazide diuretic, and kidney failure, our population showed similar results compared to ENTRED 2007-2010 [27]. Despite some difference about comorbidities like heart failure, we can consider our sample as a representative one of the French diabetic population.

\section{Conclusions}

According to this study, metformin seemed not to be associated with LA in patients with type 2 diabetes. However, in case of acute renal failure, metformin may be associated with LA. Further studies are needed to precise if metformin could have a deleterious role in other acute medical conditions. But as of now our study allows drawing the hypothesis that metformin withdrawal in case of acute intercurrent disease would probably be as important as checking contraindications before prescribing metformin in order to minimize LA risk.
Abbreviations
ACE: Angiotensin converting enzyme
AKI: Acute kidney injury
ARA: Angiotensin II receptor antagonists
ARV: Antiretroviral
CKD: Chronic kidney disease
CM: $\quad$ Contrast media
GFR: Glomerular filtration rate
ICD: International Classification of Diseases
LA: Lactic acidosis
NSAID: Nonsteroidal anti-inflammatory drugs
OR: $\quad$ Odds ratio
ROC: Receiver Operator Characteristic.

\section{Conflict of Interests}

None of the authors have a conflict of interests with respect to this study.

\section{Authors' Contribution}

Marion Lepelley performed data analysis and wrote the paper. Joris Giai and Nassima Yahiaoui performed the statistical analysis. Sébastien Chanoine contributed to the statistical analysis. Céline Villier conceived and supervised the study and contributed the paper.

\section{References}

[1] L. Guariguata, D. R. Whiting, I. Hambleton, J. Beagley, U. Linnenkamp, and J. E. Shaw, "Global estimates of diabetes prevalence for 2013 and projections for 2035," Diabetes Research and Clinical Practice, vol. 103, no. 2, pp. 137-149, 2014. 
[2] Haute Autorité de Santé-Stratégie médicamenteuse du contrôle glycémique du diabète de type 2, Août 2015, http://www .has-sante.fr/portail/jcms/c_1022476/fr/strategie-medicamenteuse-du-controle-glycemique-du-diabete-de-type- 2 .

[3] S. E. Inzucchi, R. M. Bergenstal, J. B. Buse et al., "Management of hyperglycaemia in type 2 diabetes: a patient-centered approach. Position statement of the American Diabetes Association (ADA) and the European Association for the Study of Diabetes (EASD)," Diabetologia, vol. 55, no. 6, pp. 1577-1596, 2012.

[4] R. C. Turner, C. A. Cull, V. Frighi, and R. R. Holman, "Glycemic control with diet, sulfonylurea, metformin, or insulin in patients with type 2 diabetes mellitus. Progressive requirement for multiple therapies (UKPDS 49)," The Journal of the American Medical Association, vol. 281, no. 21, pp. 2005-2012, 1999.

[5] J.-D. Lalau, "Lactic acidosis induced by metformin: incidence, management and prevention," Drug Safety, vol. 33, no. 9, pp. 727-740, 2010.

[6] V. Biradar, J. L. Moran, S. L. Peake, and J. V. Peter, "Metforminassociated lactic acidosis (MALA): clinical profile and outcomes in patients admitted to the intensive care unit," Critical Care and Resuscitation, vol. 12, no. 3, pp. 191-195, 2010.

[7] S. Salpeter, E. Greyber, G. Pasternak, and E. Salpeter, "Risk of fatal and nonfatal lactic acidosis with metformin use in type 2 diabetes mellitus," Cochrane Database of Systematic Reviews, no. 4, Article ID CD002967, 2010.

[8] M. Bodmer, C. Meier, S. Krähenbühl, S. S. Jick, and C. R. Meier, "Metformin, sulfonylureas, or other antidiabetes drugs and the risk of lactic acidosis or hypoglycemia: a nested case-control analysis," Diabetes Care, vol. 31, no. 11, pp. 2086-2091, 2008.

[9] J.-D. Lalau and J.-M. Race, "Lactic acidosis in metformintreated patients. Prognostic value of arterial lactate levels and plasma metformin concentrations," Drug Safety, vol. 20, no. 4, pp. 377-384, 1999.

[10] S. Vecchio and A. Protti, "Metformin-induced lactic acidosis: no one left behind," Critical Care, vol. 15, no. 1, article 107, 2011.

[11] I. R. F. van Berlo-van de Laar, C. G. Vermeij, and C. J. Doorenbos, "Metformin associated lactic acidosis: incidence and clinical correlation with metformin serum concentration measurements," Journal of Clinical Pharmacy and Therapeutics, vol. 36, no. 3, pp. 376-382, 2011.

[12] T. A. Chowdhury, R. Wright, and M. M. Yaqoob, "Using metformin in the presence of renal disease," The British Medical Journal, vol. 350, Article ID h1758, 2015.

[13] A. T. Calabrese, K. C. Coley, S. V. DaPos, D. Swanson, and R. H. Rao, "Evaluation of prescribing practices: risk of lactic acidosis with metformin therapy," Archives of Internal Medicine, vol. 162, no. 4, pp. 434-437, 2002.

[14] D. T. Eurich, F. A. McAlister, D. F. Blackburn et al., "Benefits and harms of antidiabetic agents in patients with diabetes and heart failure: systematic review," British Medical Journal, vol. 335, no. 7618, pp. 497-501, 2007.

[15] N. Papanas, E. Maltezos, and D. P. Mikhailidis, "Metformin and heart failure: never say never again," Expert Opinion on Pharmacotherapy, vol. 13, no. 1, pp. 1-8, 2012.

[16] K. P. Vasisht, S.-C. Chen, Y. Peng, and G. L. Bakris, "Limitations of metformin use in patients with kidney disease: are they warranted?" Diabetes, Obesity and Metabolism, vol. 12, no. 12, pp. 1079-1083, 2010.

[17] W. E. Cayley Jr., "Does metformin increase the risk of fatal or nonfatal lactic acidosis?" American Family Physician, vol. 82, no. 9, pp. 1068-1069, 2010.
[18] A. Holstein, D. Nahrwold, S. Hinze, and E.-H. Egberts, "Contraindications to metformin therapy are largely disregarded," Diabetic Medicine, vol. 16, no. 8, pp. 692-696, 1999.

[19] R. D. Cohen and H. F. Woods, "Lactic acidosis revisited," Diabetes, vol. 32, no. 2, pp. 181-191, 1983.

[20] A. Emslie-Smith, D. I. R. Boyle, J. M. M. Evans, F. Sullivan, and A. D. Morris, "Contraindications to metformin therapy in patients with type 2 diabetes-a population-based study of adherence to prescribing guidelines," Diabetic Medicine, vol. 18, no. 6, pp. 483-488, 2001.

[21] K. J. Lipska, C. J. Bailey, and S. E. Inzucchi, "Use of metformin in the setting of mild-to-moderate renal insufficiency," Diabetes Care, vol. 34, no. 6, pp. 1431-1437, 2011.

[22] G. Sterner, S. Elmståhl, and A. Frid, "Renal function in a large cohort of metformin treated patients with type 2 diabetes mellitus," British Journal of Diabetes and Vascular Disease, vol. 12, no. 5, pp. 227-231, 2012.

[23] Type 2 Diabetes, 1-Recommendations, Guidance and Guidelines, August 2015, http://www.nice.org.uk/guidance/cg87/chapter/1-recommendations.

[24] T. Scale and J. N. Harvey, "Diabetes, metformin and lactic acidosis," Clinical Endocrinology, vol. 74, no. 2, pp. 191-196, 2011.

[25] C. J. Girman, T. D. Kou, K. Brodovicz et al., "Risk of acute renal failure in patients with Type 2 diabetes mellitus," Diabetic Medicine, vol. 29, no. 5, pp. 614-621, 2012.

[26] J. K. Duong, T. J. Furlong, D. M. Roberts et al., "The role of metformin in metformin-associated lactic acidosis (MALA): case series and formulation of a model of pathogenesis," Drug Safety, vol. 36, no. 9, pp. 733-746, 2013.

[27] InVS-BEH no. 42-43, Diabète traité: quelles évolutions entre 2000 et 2009 en France ?, Disponible sur, 2010, http://www.invs .sante.fr/beh/2010/42_43/. 


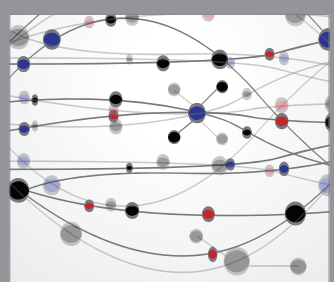

The Scientific World Journal
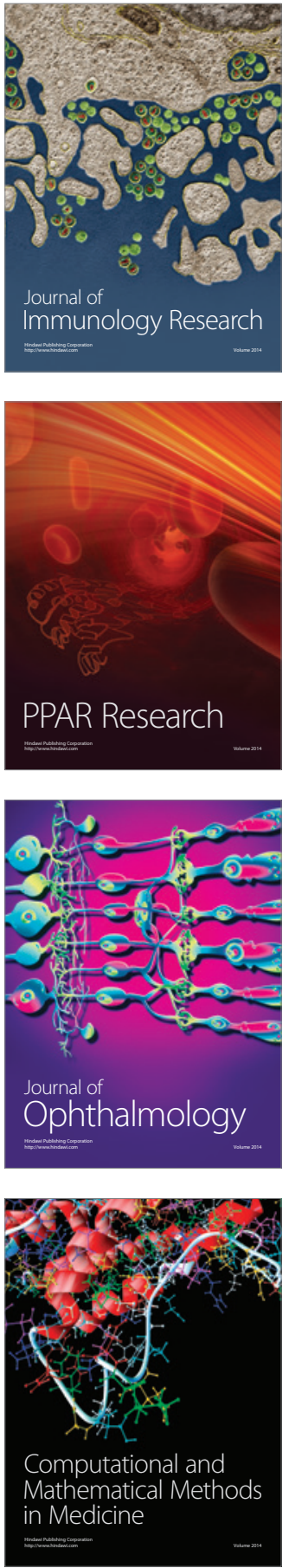

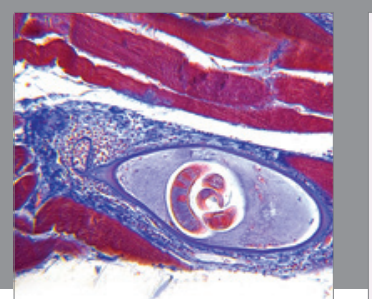

Gastroenterology Research and Practice

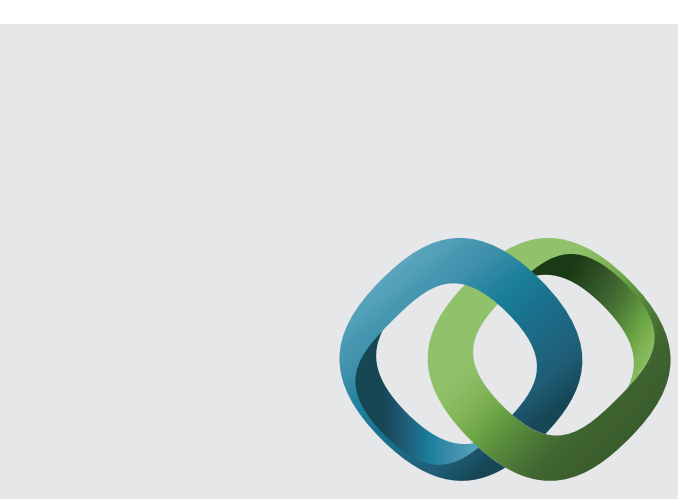

\section{Hindawi}

Submit your manuscripts at

http://www.hindawi.com
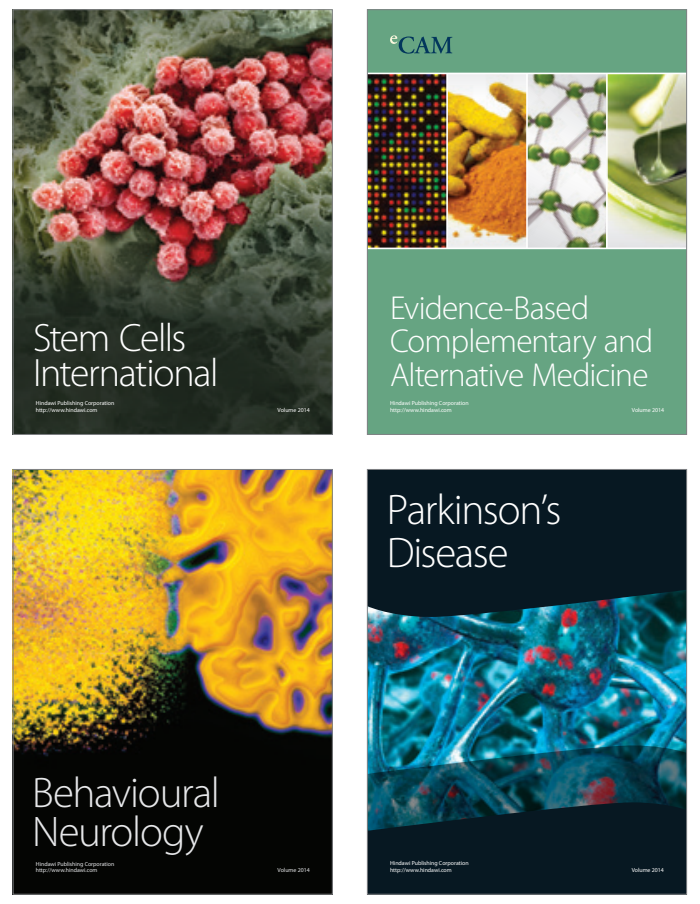
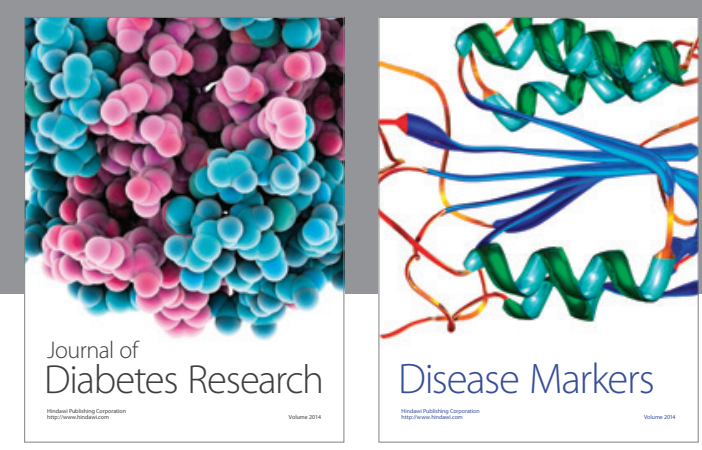

Disease Markers
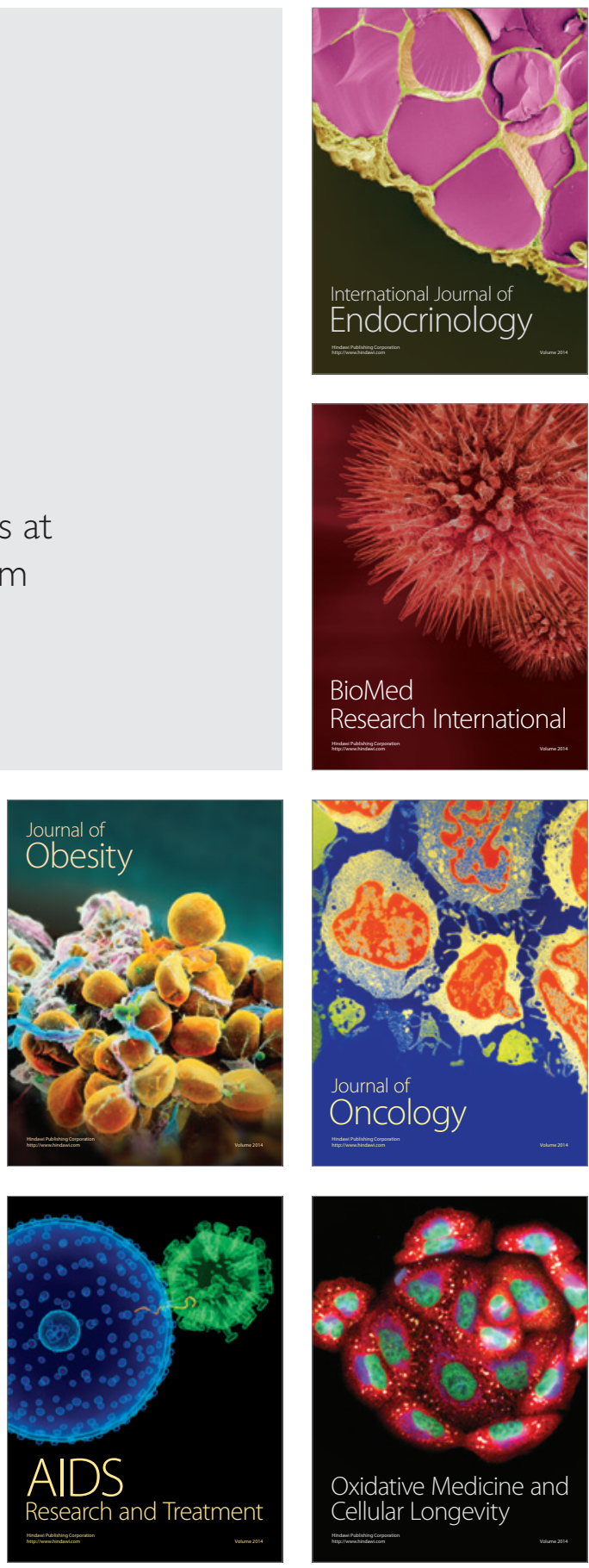